Research Article

\title{
Risk of Uterine Rupture with Vaginal Birth after Cesarean in Twin Gestations
}

\author{
Kimya Baradaran (iD \\ Master of Science in Physician Assistant Studies, Dominican University of California, San Rafael, CA 94901, USA \\ Correspondence should be addressed to Kimya Baradaran; kimya.baradaran@students.dominican.edu
}

Received 29 November 2020; Accepted 24 March 2021; Published 1 April 2021

Academic Editor: Ixora Kamisan Atan

Copyright (C) 2021 Kimya Baradaran. This is an open access article distributed under the Creative Commons Attribution License, which permits unrestricted use, distribution, and reproduction in any medium, provided the original work is properly cited.

Background. Women with a previous cesarean delivery may attempt a subsequent vaginal birth or repeat cesarean. Vaginal birth after cesarean carries a greater risk of uterine rupture, defined as the disruption of all uterine layers, resulting in maternal-fetal morbidity or mortality. It is unclear how the risk of uterine rupture compares in patients with twin gestations who undergo different delivery methods. Objective. The purpose of this systematic review is to determine if there is an increased risk of uterine rupture in patients with twin gestations attempting vaginal birth after cesarean (VBAC) versus planned repeat cesarean delivery (PRCD). Study Design. PubMed, Cochrane Library, and CINAHL were searched systematically. Eligible studies were prospective and retrospective studies that evaluated the incidence of uterine rupture in twin pregnancies that attempted VBAC or PRCD. Data were manually extracted from these studies, and the number of events in each group was used to calculate an odds ratio (OR) and 95\% confidence interval (CI). Results. 4 retrospective studies were included with a total of 7699 participants, 2305 of whom attempted VBAC and 5394 underwent PRCD. The absolute risk of uterine rupture in the VBAC and PRCD groups was $0.87 \%$ and $0.09 \%$, respectively. The rate of uterine rupture was significantly higher in the VBAC group than in the PRCD group (OR: 9.43; CI: 3.54-25.17). Conclusion. Although VBAC is associated with higher rates of uterine rupture in twin pregnancies when compared with PRCD, the absolute risk of uterine rupture is low in both groups. Depending on individual risk factors, vaginal birth may be offered as a safe option to women with twin pregnancies and a history of cesarean delivery.

\section{Introduction}

The rate of cesarean deliveries in the United States has increased significantly, from $5.5 \%$ in 1970 to $31.9 \%$ in 2016 [1]. Similarly, the national rate of twin deliveries has increased 79\% from 1980 to 2016 (from 18.9 to 33.9 per 1,000) [2]. As a result, obstetricians are encountering more patients with both twin gestations and a history of cesarean. Multiple pregnancies (97-98\% of which are twins) have a twofold risk of maternal death and more complications including eclampsia, postpartum hemorrhage, and preterm labor [3-5]. Women with a previous cesarean have a greater risk of placental issues and twice the risk of maternal morbidity, which increases progressively as the number of previous cesareans increases [6-10].

Women with a previous cesarean have the option of attempting vaginal birth after cesarean (VBAC) or a planned repeat cesarean delivery (PRCD) in a subsequent pregnancy [11]. Overall, 73.6\% of VBAC attempts result in a successful vaginal delivery [12]. The likelihood of achieving VBAC varies based on demographic and obstetric characteristics. Risk factors for failed VBAC include maternal age $\geq 35$, maternal body mass index $\geq 30$, birth weight $>4000 \mathrm{~g}$, and gestational age $>40$ weeks at delivery [12-16]. Evidence also shows that labor induction or augmentation with oxytocin reduces the chance of a successful VBAC when compared to spontaneous labor without augmentation [12]. Compared to PRCD, VBAC attempts have higher rates of endometritis, respiratory distress syndrome, and uterine rupture, but lower rates of hysterectomy and wound complications, shorter recovery periods, and less blood loss [11, 17-19].

Uterine rupture is defined as a complete disruption of all uterine layers, including the serosa, resulting in a change in maternal or fetal status [20]. The incidence of uterine 
rupture is $0.4-0.7 \%$ in patients who attempt VBAC, but this risk is higher with increased maternal and gestational age and induction with oxytocin [21-25]. Fetal complications of uterine rupture include hypoxic-ischemic encephalopathy, impaired motor development, and death, while maternal complications include postpartum hemorrhage, hysterectomy, genitourinary injury, and death $[21,22,24,26]$. Evidently, uterine rupture, while rare, carries a high risk of maternal-fetal morbidity and mortality. Various studies have shown null and positive associations between VBAC attempts in twin pregnancies and the risk of uterine rupture as compared to PRCD [27-34]. Due to this controversy in the literature, there is a need for a systematic review. This systematic review aims to determine the risk of uterine rupture with VBAC attempts versus PRCD in patients with twin gestations. The results of this study will aid in clinical decision-making when recommending patients with a history of cesarean to deliver twins vaginally versus via planned cesarean.

\section{Methods}

The current systematic review was conducted in accordance with the Preferred Reporting Items for Systematic Reviews and Meta-Analysis (PRISMA) guidelines. A systematic manual search of major databases was conducted in PubMed, Cochrane Library, and CINAHL to identify all prospective observational studies and retrospective cohort studies comparing VBAC attempt and PRCD in twin gestations. The search was completed from inception to September 2018 without any language restrictions. The PICO (patient, intervention, comparator, and outcome) statement was used to perform the literature search. Search terms were related to the population of interest (women with twin pregnancies and a previous cesarean), intervention (VBAC attempt), comparator (PRCD), and outcome (uterine rupture). The following keywords were used: twin, trial of labor, vaginal birth after cesarean, previous cesarean, and repeat cesarean. The references of the included studies and prior reviews on the same topic were also screened to identify additional relevant articles. A stepwise approach was utilized for selecting the final studies.

All records were manually screened by title and abstract to ensure that they aligned with the population, exposure, and outcome of this study. Studies that were potential candidates were further evaluated using the inclusion and exclusion criteria. A study was included if it was prospective or retrospective, considered women with twin pregnancies and a previous cesarean, compared VBAC attempt and PRCD, and assessed for uterine rupture. Studies were excluded if they were reviews, commentaries, or case reports, not written in English, did not report any cases of uterine rupture in either group, or reported uterine dehiscence. Lastly, the qualities of the potential studies were rated using the Newcastle-Ottawa Scale (NOS). Studies were included if they received a fair or good rating, but were eliminated if they received a poor rating (defined as $0-1$ stars in the selection domain, 0 stars in the compatibility domain, or 0-1 stars in the exposure/outcome domain). Clinically relevant data were extracted regarding study year, study design, and study period, single versus multicenter study, total number of participants, number of participants in each group, and number of events in each group. Since the outcome was not present in every group, the sum of the events across the studies was used to calculate an odds ratio (OR) and $95 \%$ confidence interval (CI).

\section{Results}

A total of 712 records were screened and 19 full-text articles were evaluated. 15 studies were eliminated based on exclusion criteria. 4 studies were considered potentially eligible, and none of them were determined to be of poor quality (Figure 1).

A total of 4 retrospective studies originating from the United States and published between 1996 and 2006 were included in the systematic review [31-34]. 3 of the studies gathered data from multiple medical centers [32-34], while 1 study obtained data from a single hospital [31]. Collectively, the studies identified 7699 women with twin pregnancies and a previous cesarean. Of these, 2305 attempted VBAC and 5394 underwent PRCD (Table 1).

Uterine rupture rates ranged from $0 \%$ to $1.69 \%$ (Table 2). Of the 4 studies, 3 found no significant difference in uterine rupture rates between the groups [31-33], while the largest study reported an increased rate of uterine rupture with VBAC attempts [34].

\section{Discussion}

Systematic review of published studies revealed that the risk of uterine rupture is significantly higher in women with twin gestations who attempt VBAC as opposed to PRCD. However, the absolute risk of uterine rupture is low in both groups, as shown by the low percentages of uterine rupture (Table 2) and the fact that 3 out of 4 studies contained a group with 0 cases of uterine rupture [31-33]. Notably, the study with the largest patient population reported cases of uterine rupture in both groups and demonstrated a significantly greater risk of uterine rupture in the VBAC group [34]. Meanwhile, the other 3 studies found no significant difference between rates of uterine rupture among the groups [31-33]. Nevertheless, this study shows that electing to have a PRCD reduces but does not eliminate the small risk of uterine rupture.

\section{Conclusion}

This review determined that women with twin gestations and a previous cesarean delivery are at a higher risk of uterine rupture from vaginal delivery versus another cesarean. This information should be provided during prenatal counseling to help guide clinical decisions. Despite this, clinicians should be cautious not to develop an overall perception of high risk regarding VBAC in twin pregnancies. Clinicians should consider that the increased risk of uterine rupture is statistically but not necessarily clinically significant. Clinicians should discuss with their patients the option 


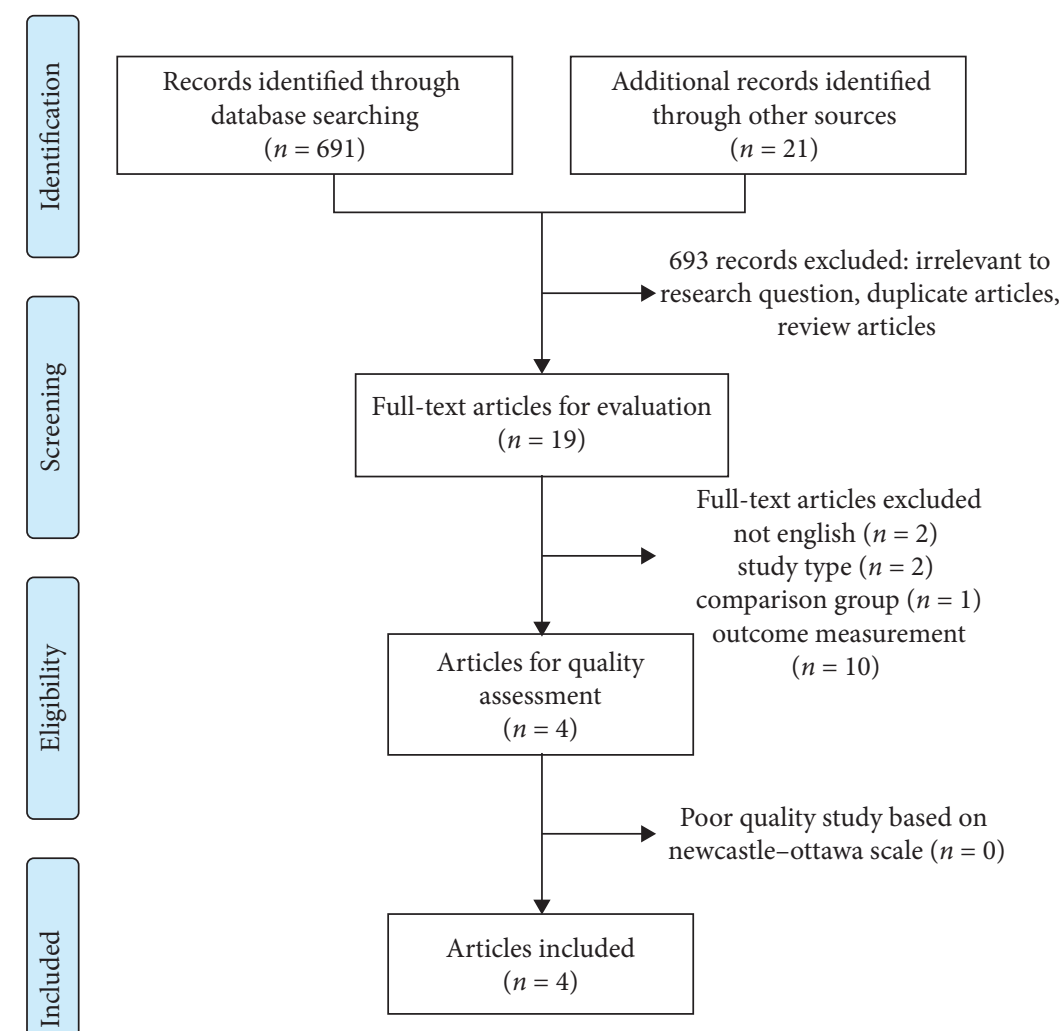

Figure 1: Study selection process.

TABLe 1: Baseline study characteristics [31-34].

\begin{tabular}{|c|c|c|c|c|c|c|}
\hline Study and year & Study design & Study period & $\begin{array}{c}\text { Single center or } \\
\text { multicenter }\end{array}$ & $\begin{array}{c}\text { Number of } \\
\text { patients }\end{array}$ & Number of VBAC & Number of PRCD \\
\hline Miller et al., 1996 & Retrospective & 1985-1994 & Single center & 210 & 92 & 118 \\
\hline Cahill et al., 2005 & Retrospective & $1996-2000$ & Multicenter & 522 & 177 & 345 \\
\hline Varner et al., 2005 & Retrospective & 1999-2002 & Multicenter & 412 & 186 & 226 \\
\hline Ford et al., 2006 & Retrospective & 1993-2002 & Multicenter & 6555 & 1850 & 4705 \\
\hline Total & & & & 7699 & 2305 & 5394 \\
\hline
\end{tabular}

TABLE 2: Study outcomes [31-34].

\begin{tabular}{|c|c|c|c|c|c|c|c|}
\hline \multirow{2}{*}{ Study } & \multicolumn{3}{|c|}{ VBAC attempt } & \multicolumn{3}{|c|}{ PRCD } & \multirow{2}{*}{ OR, 95\% CI } \\
\hline & Events & Total & Percent & Events & Total & Percent & \\
\hline Miller et al. [31] & 0 & 92 & 0 & 2 & 118 & 1.69 & \\
\hline Cahill et al. [32] & 2 & 177 & 1.13 & 0 & 345 & 0 & \\
\hline Varner et al. [33] & 2 & 186 & 1.08 & 0 & 226 & 0 & \\
\hline Ford et al. [34] & 16 & 1850 & 0.86 & 3 & 4705 & 0.06 & \\
\hline Total & 20 & 2305 & 0.87 & 5 & 5394 & 0.09 & $9.43,[3.54-25.17]$ \\
\hline
\end{tabular}

of attempting a VBAC, especially if the patient is free of additional risk factors that increase the rate of uterine rupture.

Since the data for this systematic review were obtained from multicenter studies, the results can be generalized to a broad patient population. Nevertheless, this study has some inherent limitations. First, there exists the possibility of selection bias since retrospective studies were used. Second, the number of previous cesarean or vaginal deliveries was not considered in this study, so the results may not be validated for those with a history of multiple cesareans or no prior vaginal deliveries. Last, this study focused on the risk of uterine rupture and did not consider other adverse maternal and fetal outcomes such as hemorrhage or infection, which can alter the risk-benefit ratio of each situation. 
In conclusion, while the relative risk of uterine rupture is higher for VBAC attempts, the absolute risk is low so VBAC may be considered a safe and effective option in many women with twins. An individualized approach must be used to consider other risk factors, such as maternal and gestational age, that may affect the outcome of delivery. Clinicians must also consider and discuss maternal-fetal risks other than uterine rupture when determining the safest delivery method for a patient. Thus, the option of VBAC may be safely offered to women with twin gestations and a history of cesarean depending on their additional risk factors.

\section{Data Availability}

The data used to support the findings of this study are included within the article.

\section{Disclosure}

This manuscript has been presented in Dominican Scholar as part of educational requirements for the MSPAS program and can be accessed via the following link: https://scholar. dominican.edu/cgi/viewcontent.cgi?

article $=1009 \&$ context $=$ physician-assistant-studies-student articles.

\section{Conflicts of Interest}

The author declares that there are no conflicts of interest regarding the publication of this article.

\section{References}

[1] P. J. Placek and S. M. Taffel, "Trends in cesarean section rates for the United States, 1970-78," Obstetrical \& Gynecological Survey, vol. 36, no. 8, pp. 433-434, 1981.

[2] J. A. Martin, B. E. Hamilton, M. J. K. Osterman, A. K. Driscoll, and P. Drake, "Births: final data for 2016," National Vital Statistics Report, vol. 67, no. 1, p. 7, 2018.

[3] S. P. Chauhan, J. A. Scardo, E. Hayes, A. Z. Abuhamad, and V. Berghella, "Twins: prevalence, problems, and preterm births," American Journal of Obstetrics and Gynecology, vol. 203, no. 4, pp. 305-315, 2010.

[4] A. Conde-Agudelo, J. M. Belizán, and G. Lindmark, "Maternal morbidity and mortality associated with multiple gestations," Obstetrics \& Gynecology, vol. 95, no. 6, pp. 899-904, 2000.

[5] E. R. Norwitz, V. Edusa, and J. S. Park, "Maternal physiology and complications of multiple pregnancy," Seminars in Perinatology, vol. 29, no. 5, pp. 338-348, 2005.

[6] A. K. Daltveit, M. C. Tollånes, H. Pihlstrøm, and L. M. Irgens, "Cesarean delivery and subsequent pregnancies," Obstetrics \& Gynecology, vol. 111, no. 6, pp. 1327-1334, 2008.

[7] K. E. Gray, E. R. Wallace, K. R. Nelson, S. D. Reed, and M. A. Schiff, "Population-based study of risk factors for severe maternal morbidity," Paediatric and Perinatal Epidemiology, vol. 26, no. 6, pp. 506-514, 2012.

[8] J. M. Guise, K. Eden, C. Emeis et al., "Vaginal birth after cesarean: new insights," Evidence Reports/technology Assessments, vol. 191, pp. 1-397, 2010.

[9] N. E. Marshall, R. Fu, and J.-M. Guise, "Impact of multiple cesarean deliveries on maternal morbidity: a systematic review," American Journal of Obstetrics and Gynecology, vol. 205, no. 3, p. e1, 2011.

[10] R. M. Silver, M. B. Landon, D. J. Rouse et al., "Maternal morbidity associated with multiple repeat cesarean deliveries," Obstetrics \& Gynecology, vol. 107, no. 6, pp. 1226-1232, 2006.

[11] Moawad, "Women's health care physicians," 2017, https:// www.acog.org/Patients/FAQs/Vaginal-Birth-After-CesareanDelivery.

[12] M. B. Landon, S. Leindecker, C. Y. Spong et al., "The MFMU Cesarean Registry: factors affecting the success of trial of labor after previous cesarean delivery," American Journal of $\mathrm{Ob}$ stetrics and Gynecology, vol. 193, no. 3, pp. 1016-1023, 2005.

[13] C. M. Zelop, T. D. Shipp, A. Cohen, J. T. Repke, and E. Lieberman, "Trial of labor after 40 Weeks' gestation in women with prior cesarean," Obstetrics \& Gynecology, vol. 97, no. 3, pp. 391-393, 2001.

[14] C. M. Zelop, T. D. Shipp, J. T. Repke, A. Cohen, and E. Lieberman, "Outcomes of trial of labor following previous cesarean delivery among women with fetuses weighing $>4000$ g," American Journal of Obstetrics and Gynecology, vol. 185, no. 4, pp. 903-905, 2001.

[15] S. K. Srinivas, D. M. Stamilio, M. D. Sammel et al., "Vaginal birth after caesarean delivery: does maternal age affect safety and success?" Paediatric and Perinatal Epidemiology, vol. 21, no. 2, pp. 114-120, 2007.

[16] P. T. Goodall, J. T. Ahn, J. B. Chapa, and J. U. Hibbard, "Obesity as a risk factor for failed trial of labor in patients with previous cesarean delivery," American Journal of Obstetrics and Gynecology, vol. 192, no. 5, pp. 1423-1426, 2005.

[17] S. C. Curtin, K. D. Gregory, L. M. Korst, and S. F. Uddin, "Maternal morbidity for vaginal and cesarean deliveries, according to previous cesarean history: new data from the birth certificate," National Vital Statistics Report, vol. 64, no. 4, p. 5, 2015.

[18] S. A. Gilbert, W. A. Grobman, M. B. Landon et al., "Elective repeat cesarean delivery compared with spontaneous trial of labor after a prior cesarean delivery: a propensity score analysis," American Journal of Obstetrics and Gynecology, vol. 206, no. 4, 2012.

[19] A. C. Varner and V. D’Addario, "Maternal morbidity following a trial of labor after cesarean section vs elective repeat cesarean delivery: a systematic review with metaanalysis," American Journal of Obstetrics and Gynecology, vol. 199, no. 3, pp. 224-231, 2008.

[20] M. B. Landon and H. Frey, "Uterine rupture: after previous cesarean delivery," 2019, https://www.uptodate.com/ contents/uterine-rupture-after-previous-cesarean-delivery.

[21] I. Al-Zirqi, B. Stray-Pedersen, L. Forsén, and S. Vangen, "Uterine rupture after previous caesarean section," BJOG: An International Journal of Obstetrics \& Gynaecology, vol. 117, no. 7, pp. 809-820, 2010.

[22] S. P. Chauhan, J. N. Martin, C. E. Henrichs, J. C. Morrison, and E. F. Magann, "Maternal and perinatal complications with uterine rupture in 142,075 patients who attempted vaginal birth after cesarean delivery: a review of the literature," American Journal of Obstetrics and Gynecology, vol. 189, no. 2, pp. 408-417, 2003.

[23] C. Holmgren, J. R. Scott, T. F. Porter, M. S. Esplin, and T. Bardsley, "Uterine rupture with attempted vaginal birth after cesarean delivery," Obstetrics \& Gynecology, vol. 119, no. 4, pp. 725-731, 2012.

[24] M. B. Landon, J. C. Hauth, K. J. Leveno et al., "Varner MW national institute of child health and human development 
maternal-fetal medicine units network." New England Journal of Medicine, vol. 351, no. 25, pp. 2581-2589, 2004.

[25] C. M. Zelop, T. D. Shipp, J. T. Repke, A. Cohen, A. B. Caughey, and E. Lieberman, "Uterine rupture during induced or augmented labor in gravid women with one prior cesarean delivery," American Journal of Obstetrics and Gynecology, vol. 181, no. 4, pp. 882-886, 1999.

[26] E. Bujold and R. J. Gauthier, "Neonatal morbidity associated with uterine rupture: what are the risk factors?" American Journal of Obstetrics and Gynecology, vol. 186, no. 2, pp. 311-314, 2002.

[27] D. Aaronson, A. Harlev, E. Sheiner, and A. Levy, "Trial of labor after cesarean section in twin pregnancies: maternal and neonatal safety," The Journal of Maternal-Fetal \& Neonatal Medicine, vol. 28, pp. 1-5, 2009.

[28] T. Myles, "Vaginal birth of twins after a previous cesarean section," Journal of Maternal-Fetal and Neonatal Medicine, vol. 10, no. 3, pp. 171-174, 2001.

[29] T. Myles and R. Miranda, "Vaginal birth after cesarean delivery in the twin gestation," Obstetrics \& Gynecology, vol. 95, no. 4, p. S65, 2000.

[30] A. Sansregret, E. Bujold, and R. J. Gauthier, "Twin delivery after a previous caesarean: a twelve-year experience," Journal of Obstetrics and Gynaecology Canada, vol. 25, no. 4, pp. 294-298, 2003.

[31] D. A. Miller, P. Mullin, D. Hou, and R. H. Paul, "Vaginal birth after cesarean section in twin gestation," American Journal of Obstetrics and Gynecology, vol. 175, no. 1, pp. 194-198, 1996.

[32] A. Cahill, D. M. Stamilio, E. Paré et al., "Vaginal birth after cesarean (VBAC) attempt in twin pregnancies: is it safe?" American Journal of Obstetrics and Gynecology, vol. 193, no. 3, pp. 1050-1055, 2005.

[33] M. W. Varner, S. Leindecker, C. Y. Spong et al., "The Maternal-Fetal Medicine Unit cesarean registry: trial of labor with a twin gestation," American Journal of Obstetrics and Gynecology, vol. 193, no. 1, pp. 135-140, 2005.

[34] A. A. D. Ford, B. T. Bateman, and L. L. Simpson, "Vaginal birth after cesarean delivery in twin gestations: a large, nationwide sample of deliveries," American Journal of Obstetrics and Gynecology, vol. 195, no. 4, pp. 1138-1142, 2006. 